\title{
Risk factors for and clinical outcomes of carbapenem non-susceptible gram negative bacilli bacteremia in patients with acute myelogenous leukemia
}

Dong Hoon Shin ${ }^{1 \dagger}$, Dong-Yeop Shin ${ }^{1,2+}$, Chang Kyung Kang ${ }^{1 *}$ (D), Suhyeon Park ${ }^{1,2}$, Jieun Park ${ }^{1,2}$, Kang II Jun , Taek Soo Kim ${ }^{3}$, Youngil Koh ${ }^{1,2}$, Jun Shik Hong ${ }^{1,2}$, Pyoeng Gyun Choe', Wan Beom Park, Nam-Joong Kim', Sung-soo Yoon ${ }^{1,2}$, Inho Kim ${ }^{1,2^{*}}$ and Myoung-don $\mathrm{Oh}^{1}$

\begin{abstract}
Background: Carbapenem is frequently used when gram negative bacilli (GNB) bacteremia is detected especially in neutropenic patients. Consequently, appropriate treatment could be delayed in GNB bacteremia cases involving organisms which are not susceptible to carbapenem (carba-NS), resulting in a poor clinical outcomes. Here, we explored risk factors for carba-NS GNB bacteremia and its clinical outcomes in patients with acute myelogenous leukemia (AML) that underwent chemotherapy.

Methods: We reviewed all GNB bacteremia cases that occurred during induction or consolidation chemotherapy, over a 15-year period, in a tertiary-care hospital.

Results: Among 489 GNB bacteremia cases from 324 patients, 45 (9.2\%) were carba-NS and 444 (90.8\%) were carbapenem susceptible GNB. Independent risk factors for carba-NS GNB bacteremia were: carbapenem use at bacteremia onset (adjusted odds ratio [aOR]: 91.2; 95\% confidence interval [95\%Cl]: 29.3-284.1; $P<0.001$ ); isolation of carbapenem-resistant Acinetobacter baumannii (aOR: 19.4, 95\%Cl: 3.4-112.5; $P=0.001$ ) in the prior year; and days from chemotherapy to GNB bacteremia (aOR: 1.1 per day, 95\%Cl: 1.1-1.2; $P<0.001$ ). Carba-NS bacteremia was independently associated with in-hospital mortality (aOR: 6.6, 95\%Cl: 3.0-14.8; P $<0.001$ ).
\end{abstract}

Conslusion: Carba-NS organisms should be considered for antibiotic selection in AML patients having these risk factors.

Keywords: Carbapenem non-susceptible, Gram negative bacilli, Bacteremia, Acute myelogenous leukemia, Risk factors

\section{Background}

Bloodstream infection is a common and serious infectious complication in patients with hematologic malignancy, having the crude mortality rate of $12-42 \%[1-3]$. Above all, bloodstream infections caused by gram negative bacilli

\footnotetext{
* Correspondence: zeptemiger@hanmail.net; ihkimmd@snu.ac.kr

${ }^{\dagger}$ Dong Hoon Shin and Dong-Yeop Shin contributed equally to this work. 'Department of Internal Medicine, Seoul National University College of Medicine, Daehak-ro 101, Jongro-gu, Seoul 03080, Republic of Korea Full list of author information is available at the end of the article
}

(GNB) are a major cause of morbidity and mortality in patients with acute myelogenous leukemia (AML) that undergo induction or consolidation chemotherapy $[4,5]$. Patients with AML typically experience a prolonged period of neutropenia, which increases the risk of bacteremia and leads to poor outcome $[3,5]$. Since inappropriate empiric treatment is a well-known risk factor for a fatal outcome [6-8], early administration of an effective antibiotic is crucial to treat this deadly condition.

C C The Author(s). 2020 Open Access This article is licensed under a Creative Commons Attribution 4.0 International License, which permits use, sharing, adaptation, distribution and reproduction in any medium or format, as long as you give appropriate credit to the original author(s) and the source, provide a link to the Creative Commons licence, and indicate if changes were made. The images or other third party material in this article are included in the article's Creative Commons licence, unless indicated otherwise in a credit line to the material. If material is not included in the article's Creative Commons licence and your intended use is not permitted by statutory regulation or exceeds the permitted use, you will need to obtain permission directly from the copyright holder. To view a copy of this licence, visit http://creativecommons.org/licenses/by/4.0/ The Creative Commons Public Domain Dedication waiver (http://creativecommons.org/publicdomain/zero/1.0/) applies to the data made available in this article, unless otherwise stated in a credit line to the data. 
Carbapenems are frequently used as an empirical treatment for a broad spectrum of bacteria in patients that exhibit clinical deterioration in febrile neutropenia. Consequently, appropriate treatment might be delayed when pathogens are not susceptible to carbapenem (carba-NS), which could lead to a poor clinical outcome $[9,10]$. A few studies have investigated risk factors for carba-NS GNB bacteremia and its clinical outcomes $[9,10]$. However, no study has elucidated risk factors in patients with AML that receive chemotherapy.

We aimed to explore the risk factors for carba-NS GNB bacteremia and its clinical outcomes in patients that underwent induction or consolidation chemotherapy for AML.

\section{Methods}

\section{Study design}

This retrospective case-control study, conducted in Seoul National University Hospital, included all adult patients (aged $\geq 18$ years) with AML that contracted GNB bacteremia during induction or consolidation chemotherapy, from January 2000 to December 2015. Clinical and microbiological characteristics were compared between cases of carbapenem susceptible (carba-S) and cases of carba-NS GNB bacteremia. We also examined the association between carba-NS and in-hospital mortality.

\section{Clinical characteristics}

We retrieved clinical data from electronic medical records related to patient characteristics, including: age, sex, purpose of chemotherapy (induction or consolidation), history of diabetes mellitus, history of a resistant organism colonization in the preceding year (namely, vancomycin-resistant enterococci (VRE), extendedspectrum $\beta$-lactamase-producing enterobacteriaceae (ESBL), carbapenem-resistant Pseudomonas aeruginosa, and carbapenem-resistant Acinetobacter baumannii (CRAB)), history of GNB bacteremia in the prior year, primary site of infection, the acute severity of sepsis measured with the Pitt bacteremia score [11, 12], the presence of septic shock, antibiotics used at bacteremia onset and those used empirically before the antibiotic susceptibility results become available, and the hospitalization duration before the GNB bacteremia onset. We also collected data on all-cause mortality within 30 days after bacteremia onset, in-hospital mortality, and GNB-bacteremia-attributed mortality.

\section{Definitions}

GNB bacteremia was defined as the isolation of a GNB in at least one blood culture bottle [13]. All kinds of blood GNB isolates were regarded as pathogens. When multiple bacteremia events had occurred in a patient during a single chemotherapy treatment, each bacteremia caused by a different organism was considered a different case. Antibiotic treatments were categorized into four groups: none; antibiotics without antipseudomonal activity; antipseudomonal agents, but not carbapenem; and carbapenem. For example, non-antipseudomonal agents included cefazolin or ceftriaxone, and antipseudomonal antibiotics that were not carbapenem included piperacillin/tazobactam or cefepime. Empirical antibiotics were considered inappropriate, when the isolate was not susceptible to the selected antibiotics in vitro. Septic shock was defined as sepsis associated with systolic blood pressure below $90 \mathrm{mmHg}$ or $40 \mathrm{mmHg}$ below the baseline systolic blood pressure; or a requirement for a vasopressor to maintain blood pressure, despite adequate fluid resuscitation [7]. GNB bacteremia-attributed mortality was defined as positive blood culture for GNB at the time of death or a persistent focus of GNB infection associated with clinical signs of sepsis $[14,15]$.

\section{Microbiological analyses}

BacT/Alert FA and FN bottles (bioMe'rieux, Durham, North Carolina) were used for all blood cultures. Antimicrobial susceptibility was identified with VITEK 2 (bioMe'rieux, Marcy L'etoil, France), according to the criteria provided in the guidelines of the Clinical and Laboratory Standards Institute [16]. A bacterium was regarded as a carba-NS strain when it showed intermediate susceptibility or resistance to at least one carbapenem in vitro [16].

\section{Sensitivity analyses}

Sensitivity analyses were performed to account for potential confounders of risk factors for carba-NS GNB bacteremia or in-hospital mortality. These subgroup analyses were performed after excluding breakthrough GNB bacteremia cases that occurred during carbapenem use, since carbapenem use had been suspected to be strongly associated with the occurrence of carba-NS GNB bacteremia. Risk factors for carba-NS GNB bacteremia and those for in-hospital mortality were examined in the subgroup similar to the methods described above.

\section{Statistical analysis}

The Student's $t$ test was used to compare continuous variables, and the chi-squared test or Fisher's exact test were used to compare categorical variables. All the variables were included in the multivariate analysis using a backward stepwise logistic regression, with $P=0.05$ as the cut-off value for removing variables. Among parameters that represented the length of hospital stay and acute severity of infection, only a time interval between starting date for chemotherapy and the date of positive blood culture for GNB and Pitt bacteremia score were 
Table 1 Clinical characteristics of patients with carba-S and carba-NS GNB bacteremia

\begin{tabular}{|c|c|c|c|}
\hline Variables & Carba-S $(n=444)$ & Carba-NS $(n=45)$ & $P$ \\
\hline Age, mean $( \pm$ SD) & $51.0( \pm 14.8)$ & $56.4( \pm 13.8)$ & 0.019 \\
\hline Male & $263(59.2)$ & $20(44.4)$ & 0.056 \\
\hline Chemotherapy & & & $<0.001$ \\
\hline Induction or re-induction & $170(38.3)$ & $33(73.3)$ & \\
\hline Consolidation & $274(61.7)$ & $12(26.7)$ & \\
\hline Diabetes mellitus & $123(27.7)$ & $13(28.9)$ & 0.866 \\
\hline \multicolumn{4}{|l|}{ Isolation of resistant organism in the prior 1 year } \\
\hline VRE & $33(7.4)$ & $11(24.4)$ & 0.001 \\
\hline ESBL & $22(5.0)$ & $7(15.6)$ & 0.011 \\
\hline CRPA & $9(2.0)$ & $2(4.4)$ & 0.268 \\
\hline CRAB & $3(0.7)$ & $6(13.3)$ & $<0.001$ \\
\hline History of GNB bacteremia in the prior 1 year & $146(32.9)$ & $21(46.7)$ & 0.063 \\
\hline Presence of preceding bacteremia during the hospitalization & $42(9.5)$ & $19(42.2)$ & $<0.001$ \\
\hline \multicolumn{4}{|l|}{ Primary foci of infection } \\
\hline Intra-abdominal infection & $67(15.1)$ & $5(11.1)$ & 0.473 \\
\hline Central line associated infection & $52(11.7)$ & $9(20.0)$ & 0.086 \\
\hline Urinary tract infection & $8(1.8)$ & $0(0.0)$ & 1.000 \\
\hline Pneumonia & $6(1.4)$ & $0(0.0)$ & 1.000 \\
\hline Others & $9(2.0)$ & $0(0.0)$ & 0.610 \\
\hline Unknown & $303(68.2)$ & $31(68.9)$ & 0.929 \\
\hline Pitt score, median (IQR) & $1.6(0.0-2.0)$ & $2.2(0.0-4.0)$ & 0.171 \\
\hline Septic shock & $92(20.7)$ & $10(22.2)$ & 0.813 \\
\hline \multicolumn{4}{|l|}{ Microorganism } \\
\hline E.coli & $196(44.1)$ & $0(0.0)$ & $<0.001$ \\
\hline Klebsiella spp. & $127(28.6)$ & $2(4.4)^{\mathrm{a}}$ & $<0.001$ \\
\hline Other enterobacteriaceae & $75(16.9)$ & $1(2.2)^{\mathrm{a}}$ & 0.010 \\
\hline Pseudomonas spp. & $36(8.1)$ & $11(24.4)^{\mathrm{b}}$ & 0.002 \\
\hline S. maltophilia & $0(0.0)$ & $23(51.1)^{c}$ & $<0.001$ \\
\hline Acinetobacter spp. & $4(0.9)$ & $10(22.2)^{d}$ & $<0.001$ \\
\hline Others & $32(7.2)$ & $3(6.7)^{\mathrm{e}}$ & 0.561 \\
\hline Antibiotics at the onset of GNB bacteremia & & & $<0.001$ \\
\hline None & $252(56.8)$ & $4(8.9)$ & \\
\hline Agents without antipseudomonal activity & $40(9.0)$ & $2(4.4)$ & \\
\hline Antipseudomonal agents, not carbapenem & $147(33.1)$ & $14(31.1)$ & \\
\hline Carbapenem & $5(1.1)$ & $25(55.6)$ & \\
\hline Inappropriate empiric antibiotics & $12(2.7)$ & $28(62.2)$ & $<0.001$ \\
\hline \multicolumn{4}{|l|}{ Hospital stay } \\
\hline Days from chemotherapy to GNB bacteremia, median (IQR) & $14(11-16)$ & $22(16-29)$ & $<0.001$ \\
\hline Hospital days to GNB bacteremia, median (IQR) & $22(14-20)$ & $39(23-53)$ & $<0.001$ \\
\hline \multicolumn{4}{|l|}{ Clinical outcomes } \\
\hline 30-day mortality & $25(5.6)$ & $16(35.6)$ & $<0.001$ \\
\hline In-hospital mortality & $42(9.5)$ & $21(46.7)$ & $<0.001$ \\
\hline
\end{tabular}

Carba-S carbapenem susceptible, Carba-NS carbapenem non-susceptible, $S D$ standard deviation, VRE vancomycin resistant enterococci, ESBL extended-spectrum $\beta$ lactamase-producing enterobacteriaceae, CRPA carbapenem resistant Pseudomonas aeruginosa, CRAB carbapenem resistant Acinetobacter baumannii, GNB gram negative bacilli, $I Q R$ interquartile range

a One case was mixed GNB bacteremia case

${ }^{\mathrm{b}}$ Three cases were mixed GNB bacteremia cases

${ }^{\mathrm{C}}$ Eight cases were mixed GNB bacteremia cases

d Two cases were mixed GNB bacteremia cases

e Namely, two and one cases were Elizabethkingia meningoseptica, and Burkholderia cepacia, respectively 
included in the multivariate analysis to avoid multicollinearity, respectively. Multicollinearity was checked using variance inflation factor with 5 as the cutoff. Risk factors for in-hospital mortality or GNB bacteremia-attributed mortality were also analyzed with a multivariate regression analysis, as described above. All $P$ values $<0.05$ were considered statistically significant. All analyses were performed with PASW for Windows (version 25.0; SPSS Inc., Chicago, IL, USA).

\section{Results}

\section{GNB bacteremia cases}

During the study period, 929 patients underwent 1562 episodes of induction or consolidation chemotherapy. Among those patients, we identified 489 GNB bacteremia cases in 324 patients since 116 patients (35.8\%) suffered from GNB bacteremia more than once. Among those cases, 122 (24.9\%), 81 (16.6\%), and 286 (58.5\%) occurred during induction, re-induction, and consolidation chemotherapy, respectively. Of the total GNB bacteremia cases, 45 (9.2\%) were carba-NS GNB and 444 (90.8\%) were carba-S GNB.

\section{Clinical characteristics of patients with carba-NS GNB bacteremia}

The clinical characteristics of the two patient groups are described in Table 1. The age of patients with carba-NS GNB bacteremia was significantly higher than that of patients with carba-S GNB bacteremia (mean \pm standard deviation: $56.4 \pm 13.8$ vs. $51.0 \pm 14.8 ; P=0.019)$. Male tended to be more frequent in carba-S group (59.2\%) than in carba-NS group $(44.4 \% ; P=0.056)$. The most frequent carba-NS isolates were Stenotrophomonas maltophilia (23 cases, 51.1\%), Pseudomonas aeruginosa (11 cases, 24.4\%), and Acinetobacter baumannii (10 cases, $22.2 \%)$. Among the carba-NS GNB bacteremia cases, 10 (22.2\%) were polymicrobial infections.

Compared to the carba-S group, the carba-NS group showed significantly higher percentages of induction or re-induction chemotherapy, rather than consolidation $(P<0.001)$; isolation of VRE $(P=0.001)$, ESBL $\quad(P=$ $0.011)$, or CRAB $(P<0.001)$ in the prior year; preceding bacteremia during the hospitalization $(P<0.001)$; and carbapenem use at bacteremia onset $(P<0.001)$. In addition, the longer period from chemotherapy to bacteremia onset $(P<0.001)$, and the longer hospital stay $(P<0.001)$ were associated with carba-NS cases.

\section{Independent risk factors for carba-NS GNB bacteremia}

A multivariate logistic analysis identified three independent risk factors for carba-NS GNB bacteremia. These risk factors were: carbapenem use at the onset of bacteremia (adjusted odds ratio [aOR]: 91.2, 95\% confidence interval [95\%CI]: 29.3-284.1, $P<0.001$ ); the isolation of CRAB in the prior year (aOR: 19.4, 95\%CI: 3.4-112.5, $P=0.001) ;$ and days from chemotherapy to GNB bacteremia (aOR: 1.1 per day, 95\%CI: $1.1-1.2, P<0.001$; Table 2).

\section{Independent risk factors for mortality}

Carba-NS cases were significantly frequent in in-hospital mortality cases than in survival ones $(21 / 63,33.3 \%$ in inhospital mortality vs. $24 / 426,5.6 \%$ in survival; $P<0.001$, Table 3). Carba-NS GNB bacteremia was independently associated with in-hospital mortality (aOR: 6.6, 95\% CI: $3.0-14.8, P<0.001)$ after adjusting with induction or reinduction chemotherapy rather than consolidation (aOR: 3.5, 95\%CI: $1.7-7.0, P=0.001)$, the isolation of VRE in the prior year (aOR: 4.3, 95\%CI: $1.8-10.2, P=0.001$ ), pneumonia as a primary focus (aOR: 32.7, 95\%CI: $5.1-$ 208.5, $P<0.001$ ), or the Pitt score (aOR: 1.5 per score point, 95\%CI: $1.3-1.7, P<0.001)$.

Also, compared to the survival group, the attributed mortality group showed significantly higher percentages of carba-NS GNB bacteremia $(17 / 43,39.5 \%$ in attributed mortality vs. $24 / 426,5.6 \%$ in survival; $P<0.001$, see Additional file 1: Table S1). Carba-NS GNB bacteremia was independently associated with attributed mortality after adjusting with clinical characteristics (aOR: 9.9, 95\% CI: 3.5-27.7, $P<0.001)$.

\section{Sensitivity analysis}

To perform a subgroup analysis, we excluded all breakthrough GNB bacteremia cases that occurred during carbapenem use. Among these 459 cases, 20 (4.4\%) were carba-NS and 439 (95.6\%) were carba-S cases. Among the carba-NS cases, the most frequent carba-NS isolates were Stenotrophomonas maltophilia (8 cases, 40.0\%), Pseudomonas aeruginosa (5 cases, 25.0\%), and Acinetobacter baumannii (4 cases, 20.0\%).

Univariate and multivariate analyses were performed to elucidate independent risk factors for carba-NS GNB bacteremia in this group of patients (see Additional file 1: Table S2). Independent risk factors for carba-NS GNB

Table 2 Independent risk factors for carba-NS GNB bacteremia ${ }^{a}$

\begin{tabular}{lll}
\hline Variables & aOR $(95 \% \mathrm{Cl})$ & $P$ \\
\hline $\begin{array}{l}\text { Carbapenem use at the onset of GNB } \\
\text { bacteremia }\end{array}$ & $91.2(29.3-284.1)$ & $<0.001$ \\
$\begin{array}{l}\text { Isolation of CRAB in the prior 1 year } \\
\begin{array}{l}\text { Days from chemotherapy to GNB } \\
\text { bacteremia }\end{array}\end{array}$ & $19.4(3.4-112.5)$ & 0.001 \\
\hline
\end{tabular}

aOR adjusted odds ratio, $\mathrm{Cl}$ confidence interval, GNB gram negative bacilli, CRAB carbapenem resistant Acinetobacter baumannii

a Following variables were included in the backward stepwise logistic regression: age, induction or re-induction chemotherapy, carbapenem use at bacteremia onset, isolation of vancomycin-resistant enterococci, extendedspectrum $\beta$-lactamase-producing enterobacteriaceae, or CRAB in the prior year, preceding bacteremia during the hospitalization, and days from chemotherapy to GNB bacteremia 
Table 3 Risk factors for in-hospital mortality

\begin{tabular}{|c|c|c|c|c|c|c|}
\hline \multirow[t]{2}{*}{ Variables } & \multirow{2}{*}{$\begin{array}{l}\text { Survival } \\
(N=426)\end{array}$} & \multirow{2}{*}{$\begin{array}{l}\text { In-hospital mortality } \\
(N=63)\end{array}$} & \multicolumn{2}{|l|}{ Univariate } & \multicolumn{2}{|l|}{ Multivariate } \\
\hline & & & $\mathrm{OR}(95 \% \mathrm{Cl})$ & $P$ & aOR $(95 \% \mathrm{Cl})$ & $P$ \\
\hline Age, mean $( \pm S D)$ & $50.9( \pm 14.8)$ & $55.4( \pm 14.2)$ & $1.0(1.0-1.0)$ & 0.025 & - & - \\
\hline Male & $250(58.7)$ & $33(52.4)$ & $0.8(0.5-1.3)$ & 0.344 & - & - \\
\hline \multicolumn{7}{|l|}{ Chemotherapy } \\
\hline Induction or re-induction & $158(37.1)$ & $45(71.4)$ & $4.2(2.4-7.6)$ & $<0.001$ & $3.5(1.7-7.0)$ & 0.001 \\
\hline Consolidation & $268(62.9)$ & $18(28.6)$ & - & - & - & - \\
\hline Diabetes mellitus & $113(26.5)$ & $23(36.5)$ & $1.6(0.9-2.8)$ & 0.099 & - & - \\
\hline \multicolumn{7}{|l|}{ Isolation of resistant organism in the prior 1 year } \\
\hline VRE & $27(6.3)$ & $17(27.0)$ & $5.5(2.8-10.8)$ & $<0.001$ & $4.3(1.8-10.2)$ & 0.001 \\
\hline ESBL & $21(4.9)$ & $8(12.7)$ & $2.8(1.2-6.6)$ & 0.023 & - & - \\
\hline CRPA & $8(1.9)$ & $3(4.8)$ & $2.6(0.7-10.1)$ & 0.158 & - & - \\
\hline CRAB & $4(0.9)$ & $5(7.9)$ & $9.1(2.4-34.8)$ & 0.003 & - & - \\
\hline History of GNB bacteremia in the prior 1 year & $136(31.9)$ & $31(49.2)$ & $2.1(1.2-3.5)$ & 0.007 & - & - \\
\hline $\begin{array}{l}\text { Presence of preceding bacteremia during the } \\
\text { hospitalization }\end{array}$ & $42(9.9)$ & $19(30.2)$ & $3.9(2.1-7.4)$ & $<0.001$ & - & - \\
\hline \multicolumn{7}{|l|}{ Primary foci of infection } \\
\hline Intra-abdominal infection & $66(15.5)$ & $6(9.5)$ & $0.6(0.2-1.4)$ & 0.212 & - & - \\
\hline Central line associated infection & $54(12.7)$ & $6(9.5)$ & $0.7(0.3-1.8)$ & 0.507 & - & - \\
\hline Urinary tract infection & $7(1.6)$ & $1(1.6)$ & $1.0(1.1-8.0)$ & 1.000 & - & - \\
\hline Pneumonia & $2(0.5)$ & $4(6.3)$ & $14.4(2.6-80.2)$ & 0.003 & $32.7(5.1-208.5)$ & $<0.001$ \\
\hline Others & $9(2.1)$ & $0(0.0)$ & $0.9(0.8-0.9)$ & 0.374 & - & - \\
\hline Unknown & $288(67.6)$ & $46(73.0)$ & $1.3(0.7-2.3)$ & 0.389 & - & - \\
\hline Pitt score, median (IQR) & $1.3(0.0-2.0)$ & $4.1(1.0-5.0)$ & $1.5(1.3-1.7)$ & $<0.001$ & $1.5(1.3-1.7)$ & $<0.001$ \\
\hline Septic shock & $73(17.1)$ & $29(46.0)$ & $4.1(2.4-7.2)$ & $<0.001$ & - & - \\
\hline Carba-NS & $24(5.6)$ & $21(33.3)$ & $8.4(4.3-16.3)$ & $<0.001$ & $6.6(3.0-14.8)$ & $<0.001$ \\
\hline Inappropriate empiric antibiotics & $27(6.3)$ & $13(20.6)$ & $3.8(1.9-7.9)$ & $<0.001$ & - & - \\
\hline \multicolumn{7}{|l|}{ Hospital stay, median } \\
\hline $\begin{array}{l}\text { Days from chemotherapy to GNB bacteremia, } \\
\text { median (IQR) }\end{array}$ & $14.2(11.0-16.0)$ & $18.0(11.0-21.0)$ & $1.1(1.0-1.1)$ & $<0.001$ & - & - \\
\hline Hospital days to GNB bacteremia, median (IQR) & $21.2(14.0-20.0)$ & $40.3(18.0-61.0)$ & $1.0(1.0-1.1)$ & $<0.001$ & - & - \\
\hline
\end{tabular}

$O R$ odds ratio, $a O R$ adjusted odds ratio, $C I$ confidence interval, $S D$ standard deviation, VRE vancomycin resistant enterococci, $E S B L$ extended-spectrum $\beta$-lactamaseproducing enterobacteriaceae, CRPA carbapenem resistant Pseudomonas aeruginosa, CRAB carbapenem resistant Acinetobacter baumannii, GNB gram negative bacilli, IQR interquartile range, Carba-NS carbapenem non-susceptible

bacteremia without carbapenem exposure were the isolation of CRAB in the prior year and a time interval between chemotherapy and bacteremia. Carba-NS cases were also independently associated with in-hospital mortality in this subgroup (see Additional file 1: Table S3).

\section{Discussion}

Poor clinical outcomes are known to be associated with delayed administration of the appropriate antibiotics for GNB bacteremia in patients with AML $[7,8]$. Although carbapenems cover a broad spectrum of gram-negative bacteria, 9.2\% of GNB bacteremia cases were due to carba-NS isolates in this study. Moreover, the clinical outcomes of these cases were independently worse than the outcomes in carba-S GNB cases. We found independent risk factors for carba-NS GNB bacteremia, including carbapenem use at bacteremia onset, the isolation of CRAB in the prior year, and a prolonged time interval between chemotherapy and the onset of bacteremia.

Although carbapenem use was the most important risk factor for carba-NS GNB bacteremia, 44.4\% (20/45) of cases occurred without carbapenem administration. To reveal independent risk factors of carba-NS GNB bacteremia when carbapenems were not being administered, we performed an additional subgroup analysis. This analysis revealed that the recent isolation of a resistant organism and a prolonged interval between 
chemotherapy and bacteremia onset were independently associated with carba-NS GNB bacteremia. Therefore, when GNB bacteremia occurs in a patient with these risk factors, clinicians should consider the possibility of carba-NS pathogens, even when carbapenem was not being used.

The few previous studies that investigated risk factors for carba-NS GNB bacteremia included all hospitalized patients $[9,10]$. The first retrospective study, which was conducted in Taiwan, revealed independent risk factors for carba-NS GNB bacteremia, including previous exposure to carbapenem, and a longer hospital stay before the development of bacteremia [9]. Another case-control study showed that breakthrough GNB bacteremia during carbapenem use was significantly associated with a longer hospital stay, the presence of hematologic malignancy, and a previous colonization of causative microorganisms [10]. Although these risk factors were similar to the results of the present study, our study specifically focused on patients with AML that underwent chemotherapy.

In this study, the major pathogens identified in carbaNS GNB bacteremia were $S$. maltophilia, $P$. aeruginosa, and A. baumannii. Similar to previous studies, S. maltophilia was the most frequently identified [9, 10, 17]. Since we did not find any clinical characteristics that were independently associated with those respective organisms (data not shown), it was difficult to suggest a specific antibiotic strategy. However, in patients with GNB bacteremia that have those risk factors, especially patients that are critically ill, it would be reasonable to add trimethoprim/sulfamethoxazole and/or colistin before knowing the results of antimicrobial susceptibility tests. Further studies are warranted to elucidate whether the empirical administration of antibiotics that target carba-NS organisms might have an impact on the clinical outcomes of GNB bacteremia.

Previous studies have shown that the clinical outcome of carba-NS GNB bacteremia was worse than that of carba-S GNB bacteremia [8, 9, 18, 19]. In this study, carba-NS GNB bacteremia was independently associated with in-hospital mortality after adjusting with clinical characteristics $[18,20]$. In fact, inappropriate empirical antibiotic choices were observed significantly more often in the carba-NS group than in the carba-S group. It was previously shown that inappropriate, empirically selected antibiotic treatment was associated with in-hospital mortality [8]. Therefore, rapid antibiotic susceptibility tests that facilitate the selection of optimal agents within a short time might be an option for improving clinical outcomes of this critical condition [21, 22].

This study had some limitations. First, it was a retrospective study with a limited number of carba-NS GNB bacteremia cases. Therefore, the results should be validated in a larger prospective cohort. Second, this study was conducted in a single tertiary-care hospital. Since circulating organisms and their antimicrobial susceptibility might vary from hospital to hospital, the results of the present study should be interpreted prudently in other institutions.

\section{Conclusion}

We showed that, among patients with AML, the development of carba-NS GNB bacteremia during induction or consolidation chemotherapy was independently associated with a previous isolation of resistant organisms, a late onset of GNB bacteremia, and the use of carbapenem at onset. The clinical outcomes of carbaNS GNB bacteremia were independently worse than those of carba-S GNB bacteremia. Carba-NS GNB organisms should be considered when selecting empirical antibiotics in patients with AML that have those risk factors.

\section{Supplementary information}

Supplementary information accompanies this paper at https://doi.org/10. 1186/s12879-020-05131-2.

Additional file 1: Table S1. Risk factors for gram negative bacilli bacteremia-attributed mortality. Table S2. Risk factors for carba-NS GNB bacteremia while not using carbapenem. Table S3. Risk factors for inhospital mortality while not using carbapenem.

\section{Abbreviations}

GNB: Gram negative bacilli; AML: Acute myelogenous leukemia; CarbaNS: Carbapenem not susceptible; Carba-S: Carbapenem susceptible; VRE: Vancomycin-resistant enterococci; ESBL: Extended-spectrum $\beta$ lactamase-producing enterobacteriaceae; CRAB: Carbapenem-resistant Acinetobacter baumannii; aOR: Adjusted odds ratio; $\mathrm{Cl}$ : Confidence interval

\section{Acknowledgements}

This work was presented as an abstract (No. 116) in the IDweek, Washington, DC, USA, 2-5 October 2019.

\section{Authors' contributions}

CKK and IK conceived and designed the study; DHS, D-YS, SP, JP, KIJ, TSK, YK JSH, PGC, WBP, N-JK, S-sY, and M-dO contributed to the collection of clinical data; DHS, D-YS, CKK, WBP, and IK analyzed the data; DHS and D-YS wrote the manuscript and contributed equally to this work; CKK and IK contributed equally to this work. All authors read and approved the contents of the final version.

\section{Funding}

The authors declare that no funding has been received.

Availability of data and materials

The datasets used and/or analysed during the current study are available from the corresponding author on reasonable request.

Ethics approval and consent to participate

The study was approved by the Seoul National University Hospital Clinical Research Institute (IRB No. 1902-064-1010). Since this study did not involve experimentation on human subjects, written informed consent was waived by our institutional review board. The patient information used in this study was anonymised before its use. 


\section{Consent for publication}

Not applicable.

\section{Competing interests}

The authors declare no conflicts of interest related to this study.

\section{Author details}

'Department of Internal Medicine, Seoul National University College of Medicine, Daehak-ro 101, Jongro-gu, Seoul 03080, Republic of Korea. ${ }^{2}$ Cancer Research Institute, Seoul National University College of Medicine, Daehak-ro 101, Jongro-gu, Seoul 03080, Republic of Korea. ${ }^{3}$ Department of Laboratory Medicine, Seoul National University College of Medicine, Daehak-ro 101, Jongro-gu, Seoul 03080, Republic of Korea.

Received: 5 December 2019 Accepted: 1 June 2020

Published online: 09 June 2020

\section{References}

1. Wisplinghoff $H$, Seifert $H$, Wenzel RP, Edmond MB. Current trends in the epidemiology of nosocomial bloodstream infections in patients with hematological malignancies and solid neoplasms in hospitals in the United States. Clin Infect Dis. 2003:36:1103-10.

2. Norgaard M, Larsson H, Pedersen G, Schonheyder HC, Sorensen HT. Risk of bacteraemia and mortality in patients with haematological malignancies. Clin Microbiol Infect. 2006;12:217-23.

3. Tumbarello M, Spanu T, Caira M, Trecarichi EM, Laurenti L, Montuori E, et al. Factors associated with mortality in bacteremic patients with hematologic malignancies. Diagn Microbiol Infect Dis. 2009;64:320-6.

4. Klastersky J, de Naurois J, Rolston K, Rapoport B, Maschmeyer G, Aapro M, et al. Management of febrile neutropaenia: ESMO clinical practice guidelines. Ann Oncol. 2016;27:111-8.

5. Freifeld AG, Bow EJ, Sepkowitz KA, Boeckh MJ, Ito Jl, Mullen CA, et al. Clinical practice guideline for the use of antimicrobial agents in neutropenic patients with cancer: 2010 update by the infectious diseases society of america. Clin Infect Dis. 2011:52:56-93.

6. Metan G, Demiraslan H, Kaynar LG, Zararsiz G, Alp E, Eser B. Factors influencing the early mortality in haematological malignancy patients with nosocomial gram negative bacilli bacteraemia: a retrospective analysis of 154 cases. Braz J Infect Dis. 2013;17:143-9.

7. Angus DC, van der Poll T. Severe Sepsis and septic shock. N Engl J Med. 2013;369:840-51.

8. Kang $\mathrm{Cl}$, Kim SH, Park WB, Lee KD, Kim HB, Kim EC, et al. Bloodstream infections caused by antibiotic-resistant gram-negative bacilli: risk factors for mortality and impact of inappropriate initial antimicrobial therapy on outcome. Antimicrob Agents Chemother. 2005;49:760-6.

9. Ting SW, Lee CH, Liu JW. Risk factors and outcomes for the acquisition of carbapenem-resistant gram-negative bacillus bacteremia: a retrospective propensity-matched case control study. J Microbiol Immunol Infect. 2018;51: 621-8.

10. Lee J-Y, Kang C-I, Ko J-H, Lee WJ, Seok H-R, Park GE, et al. Clinical features and risk factors for development of breakthrough gram-negative bacteremia during Carbapenem therapy. Antimicrob Agents Chemother. 2016;60:6673-8

11. Al-Hasan MN, Lahr BD, Eckel-Passow JE, Baddour LM. Predictive scoring model of mortality in gram-negative bloodstream infection. Clin Microbiol Infect. 2013;19:948-54.

12. Battle SE, Augustine MR, Watson CM, Bookstaver PB, Kohn J, Owens WB, et al. Derivation of a quick Pitt bacteremia score to predict mortality in patients with gram-negative bloodstream infection. Infection. 2019:47:571-8.

13. Blot S, Vandewoude K, De Bacquer D, Colardyn F. Nosocomial bacteremia caused by antibiotic-resistant gram-negative Bacteria in critically ill patients: clinical outcome and length of hospitalization. Clin Infect Dis. 2002;34:1600-6.

14. Pittet D, Tarara D, Wenzel RP. Nosocomial bloodstream infection in critically III patients: excess length of stay, extra costs, and attributable mortality. JAMA. 1994;271:1598-601.

15. Harbarth S, Rutschmann O, Sudre P, Pittet D. Impact of methicillin resistance on the outcome of patients with bacteremia caused by Staphylococcus aureus. Arch Intern Med. 1998;158:182-9.

16. Melvin PW, Jean BP, April MB, Shelly C, Sharon KC, Marcelo FG, et al. M100: performance standards for antimicrobial susceptibility testing. 29th ed Wayne: Clinical and Laboratory Standards Institute; 2019.
17. Kim SH, Cho SY, Kang Cl, Seok H, Huh K, Ha YE, et al. Clinical predictors of Stenotrophomonas maltophilia bacteremia in adult patients with hematologic malignancy. Ann Hematol. 2018;97:343-50.

18. Garcia-Vidal C, Cardozo-Espinola C, Puerta-Alcalde P, Marco F, Tellez A, Aguero D, et al. Risk factors for mortality in patients with acute leukemia and bloodstream infections in the era of multiresistance. PLoS One. 2018;13: e0199531.

19. Cerceo E, Deitelzweig SB, Sherman BM, Amin AN. Multidrug-resistant gramnegative bacterial infections in the hospital setting: overview, implications for clinical practice, and emerging treatment options. Microb Drug Resist. 2016;22:412-31.

20. Halpern AB, Culakova E, Walter RB, Lyman GH. Association of Risk Factors, mortality, and care costs of adults with acute myeloid leukemia with admission to the intensive care unit. JAMA Oncol. 2017;3:374-81.

21. Kim JH, Kim TS, Song SH, Choi J, Han S, Kim DY, et al. Direct rapid antibiotic susceptibility test (dRAST) for blood culture and its potential usefulness in clinical practice. J Med Microbiol. 2018;67:325-31.

22. Kim JH, Kim TS, Jung HG, Kang CK, Jun Kl, Han S, et al. Prospective evaluation of a rapid antimicrobial susceptibility test (QMAC-dRAST) for selecting optimal targeted antibiotics in positive blood culture. J Antimicrob Chemother. 2019;74:2255-60

\section{Publisher's Note}

Springer Nature remains neutral with regard to jurisdictional claims in published maps and institutional affiliations.
Ready to submit your research? Choose BMC and benefit from:

- fast, convenient online submission

- thorough peer review by experienced researchers in your field

- rapid publication on acceptance

- support for research data, including large and complex data types

- gold Open Access which fosters wider collaboration and increased citations

- maximum visibility for your research: over $100 \mathrm{M}$ website views per year

At $\mathrm{BMC}$, research is always in progress.

Learn more biomedcentral.com/submissions 